\title{
Predicción de aparición de injuria renal aguda después de cirugía cardiovascular en la unidad de cuidados intensivos del Hospital Universitario San Ignacio*
}

\author{
Prediction of onset of acute kidney injury after \\ cardiovascular surgery at the intensive care unit of \\ Hospital San Ignacio
}

\author{
Juan Guillermo Vargas, Martha Patricia Rodríguez, \\ Paola Karina García, Álvaro Ruiz • Bogotá, D.C.
}

\section{Resumen}

Objetivo: identificar pacientes de alto riesgo de presentar injuria renal aguda en el posoperatorio de cirugía cardiovascular en la unidad de cuidados intensivos del Hospital Universitario San Ignacio.

Contexto y tipo de estudio: la población de referencia estuvo conformada por aquellos pacientes atendidos en el Hospital San Ignacio de la ciudad de Bogotá, institución de IV nivel de complejidad, con una población estudio conformada por aquellos pacientes que ingresaron a la unidad de cuidados intensivos en posoperatorio inmediato de cirugía cardiovascular.

Material y métodos: estudio observacional descriptivo, retrospectivo, tipo serie de casos. La información fue recolectada por los investigadores a partir de la revisión de las historias clínicas, es decir, a partir de una fuente secundaria. Dicha recolección se realizó teniendo en cuenta un formulario diseñado para ese fin, el cual incluye datos generales y datos de las variables de la escala de predicción, registrando los datos generales de cada uno de los pacientes, incluyendo antecedentes personales y las variables de la escala de predicción.

Durante la recolección de información se fue conformando la base de datos, la cual fue debidamente revisada y depurada, y posteriormente analizada utilizando el programa Epi Info 2000.

Resultados: se analizaron 213 pacientes que ingresaron a la unidad de cuidado intensivo del Hospital Universitario San Ignacio en el posoperatorio inmediato de CVC. Entre los pacientes analizados la incidencia de IRA fue $3.3 \%(n=7)$ los cuales fueron sometidos a TRR. Estos pacientes tuvieron una creatinina posterior al procedimiento significativamente más alta $(\mathrm{p}=0.000)$. Entre los pacientes que requirieron TRR se encontró una proporción significativamente mayor de casos con una FEVI menor a $40 \%(71.4 \%$ vs $14.1 \%)$. Otra diferencia significativa fue el porcentaje de pacientes en quienes se utilizó el balón intraaórtico, el cual fue mucho mayor entre los pacientes que recibieron TRR ( $\mathrm{p}=0.000)$. En cuanto a la relación entre la IRA y los procedimientos realizados a los pacientes se evidenció una mayor proporción de casos manejados con CEC dentro del grupo de pacientes que requirieron TRR; así mismo, la proporción de pacientes a quienes se les colocó balón intraaórtico fue significativamente mayor ( $\mathrm{p}=0.000$ ). Al aplicar la escala de riesgo en los pacientes analizados se evidenció que existían cuatro pacientes con alto riesgo de IRA cuyo puntaje fue mayor a cuatro. De estos pacientes solamente dos $(50 \%)$ terminaron en TRR.

Conclusiones: la aparición de lesión renal aguda en pacientes que son llevados a cirugía cardiovascular se ha demostrado como factor independiente de mortalidad en los pacientes en las unidades de cuidado intensivo. Los modelos predictivos para determinar la aparición de IRA en el posoperatorio de CVC han facilitado la toma de decisiones y la evaluación y estratificación del riesgo, abriendo la posibilidad de realizar intervenciones más tempranas y efectivas en pro de la recuperación, disminución de complicaciones y descenso de la morbimortalidad en estos pacientes (Acta Med Colomb 2010; 35: 166-174).

Palabras clave: falla renal aguda, cirugía cardiovascular.

\footnotetext{
*Trabajo ganador del Premio al "Mejor Trabajo de Investigación Clínica” otorgado por el XXI Congreso Colombiano de Medicina Interna, Cali, 14 al 17 de octubre de 2010.

Dr. Juan Guillermo Vargas Ángel: Médico Internista, Nefrólogo, Hospital Universitario Mayor - Mederi. Profesor Universidad del Rosario; Dra. Martha Patricia Rodríguez Sánchez: Médica Internista, Nefróloga, Hospital Universitario San Ignacio. Profesora Asistente Pontificia Universidad Javeriana; Dra. Paola Karina García Padilla: Médica Internista, Nefróloga, Jefe Unidad Renal, Hospital Universitario San Ignacio. Profesora Asistente Pontificia Universidad Javeriana; Dr. Álvaro Ruíz Morales: . Msc. FACP, Médico Internista, Epidemiólogo. Profesor Titular Pontificia Universidad Javeriana. Bogotá, D.C.

Correspondencia. Dr. Juan Guillermo Vargas

E-mail: juanguillermovargas@gmail.com Recibido: 13/VIII/10 Aceptado: 17/X/10
} 


\section{Abstract}

Objective: to identify patients at high risk for acute kidney injury in postoperative cardiovascular Objective: To identify patients at high risk for acute kidney injury in postoperative cardiovascular surgery in the Intensive Care Unit of Hospital Universitario San Ignacio.

Context and type of study: the reference population consisted of patients treated at the Hospital San Ignacio de Bogotá, institution of IV level of complexity, with a study population comprised patients admitted to the intensive care unit in the immediate postoperative period of cardiac surgery.

Materials and Methods: descriptive observational study, retrospective case series. The information was gathered by researchers from the review of medical records, that is from a secondary source. This collection is made based on a form designed for that purpose, which includes general information and data variables in the prediction, recording the general data of each patient, including personal background variables and scale prediction. During the collection of information was forming the database, which was duly revised and refined, and then analyzed using Epi Info 2000.

Results: we analyzed 213 patients admitted to the Intensive Care Unit, Hospital Universitario San Ignacio in the immediate postoperative period CVC. Among the patients analyzed the incidence of ARF was $3.3 \%(n=7)$ which were subjected to TRR. These patients had post-procedure creatinine was significantly higher $(\mathrm{p}=0.000)$. Among patients requiring RRT found a significantly higher proportion of patients with an LVEF less than $40 \%$ (71.4\% vs $14.1 \%)$. Another significant difference was the percentage of patients in whom intra-aortic balloon was used, which was much higher among patients who received RRT $(p=0.000)$. As for the relationship between the IRA and the procedures performed on patients showed a higher proportion of cases managed with $\mathrm{CPB}$ in the group of patients requiring RRT, likewise, the proportion of patients who were placed intra-aortic balloon was significantly higher $(p=0.000)$. In applying the risk score in patients analyzed showed that there were 4 patients with high risk of IRA whose score was greater than 4 . Of these patients only $2(50 \%)$ ended in TRR.

Conclusions: the occurrence of acute kidney injury in patients who are taking cardiovascular surgery has been shown as independent risk factor for mortality in patients in intensive care units. Predictive models to determine the occurrence of $\mathrm{ARF}$ in pediatric $\mathrm{CVC}$ has facilitated decision making and evaluation and risk stratification, opening the possibility of early and effective interventions towards recovery, decreased complications and decreased morbidity and mortality in these patients (Acta Med Colomb 2010; 35: 166-174).

Keywords: acute renal failure, cardiovascular surgery.

\section{Introducción}

En el posoperatorio de cirugía cardiovascular (CVC), la injuria renal aguda (IRA) se caracteriza por la incidencia variable dependiendo de la definición que se utilice y la población que se evalúe. La IRA conlleva al aumento de la morbimortalidad y de la estancia hospitalaria, así como al incremento de los costos en salud (1).

El requerimiento de terapia de reemplazo renal (TRR) después de CVC oscila entre 0.33 y $9.5 \%$.

El pronóstico de los pacientes que presentan IRA y requieren TRR después de CVC es bastante pobre. Las medidas preventivas específicas no han mostrado un claro beneficio (2). La identificación de pacientes que tienen alto riesgo de presentar IRA, ha permitido analizar mejor el comportamiento clínico y ha ofrecido la opción de adoptar nuevas medidas preventivas al igual que instaurar tratamientos específicos y tempranos para este grupo de pacientes (3).

La IRA se define como el descenso de la filtración glomerular (FG), asociado con elevación de las pruebas de función renal y disminución del gasto urinario (7). Varios investigadores han usado múltiples definiciones teniendo en cuenta cambios absolutos y relativos de la creatinina sérica. El incremento absoluto de 0.5 a $1 \mathrm{mg} / \mathrm{dL}$ o relativo de más de $25 \%$ que ocurre dentro de las primeras 24 a 72 horas se considera diagnóstico de IRA. Debido a la falta de consenso en las definiciones, la iniciativa en la calidad de diálisis aguda (ADQI) propuso el sistema "RIFLE" para definir la IRA, basándose en la severidad y los desenlaces clínicos, lo cual permite clasificar la IRA en cuatro categorías (Figura 1). Los criterios incluyen cambios en la creatinina sérica y el gasto urinario (8).

La incidencia de IRA en el posoperatorio de CVC es muy variable y depende de las características de los pacientes como la edad, la falla renal previa y las alteraciones cardiovasculares como insuficiencia cardiaca, enfermedad valvular y disfunción sistólica o diastólica del ventrículo izquierdo, que comprometan el gasto cardiaco, además del estado de hidratación, patologías asociadas, administración preoperatoria de medios de contraste y enfermedad aórtica (aneurismas, disecciones, coartación). Otros factores de riesgo son el tipo de intervención quirúrgica, la duración de la circulación extracorpórea (CEC), los tiempos de isquemia renal en la cirugía aórtica, tiempo de parada con hipotermia profunda, periodos de hipotensión intraoperatorios o requerimiento de transfusión de hemoderivados. Se han encontrado después de la cirugía complicaciones relacionadas con la 


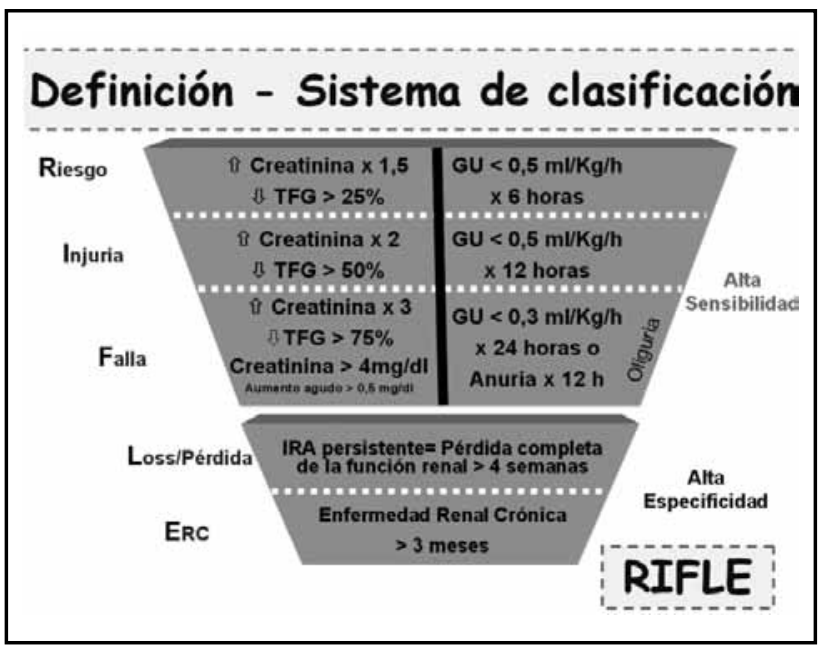

Figura 1. Clasificación RIFLE para la insuficiencia renal aguda(IRA). Tomada y adaptada de Bellomo R, Ronco C, Kellum JA, et al: Acute Dialysis Quality Initiative workgroup. Acute renal failure-Definition, outcome measures, animal models, fluid therapy and information technology needs. Crit Care 2004; 8: 204-12.

aparición de IRA tales como sepsis, estados de choque y medicamentos nefrotóxicos (9).

La incidencia de IRA en el posoperatorio de CVC con CEC oscila entre 1 y $45 \%$, (promedio 8 a $16 \%$ ) y el requerimiento de TRR entre 0.7 y $3.7 \%$ (10).

La incidencia de IRA en el posoperatorio de la cirugía de aorta, es muy variable según la localización (aorta torácica ascendente, arco aórtico, aorta torácica descendente, aorta abdominal supra o infrarrenal) y el tipo de lesión (aneurismas, disecciones, rupturas y asociación con isquemia de miembros inferiores).

La incidencia de IRA en cirugía de aneurisma de aorta torácica descendente oscila entre el 2.7 y $13.8 \%$. En la revisión de 832 pacientes operados de aneurismas de aorta torácica descendente de Svensson y Crawford (11), se observó aumento en la incidencia de IRA de $10 \%$ en disección aguda y de $15 \%$ en ruptura de aneurisma, en comparación con $6.7 \%$ cuando no hubo estos eventos. En otro estudio de Svensson en 1509 pacientes intervenidos de aneurismas de aorta toracoabdominal, la incidencia global de IRA fue de $18 \%$, siendo de $31 \%$ en los pacientes que tuvieron disección aórtica y $14 \%$ en el grupo que no la presentó. Los factores predictores para aparición de IRA encontrados en este estudio fueron edad $>70$ años, disfunción renal previa, enfermedad cerebrovascular y tiempos prolongados de isquemia renal (12).

La IRA en el posoperatorio es multifactorial, coexisten las lesiones vasculares y tubulares (13). La necrosis tubular aguda (NTA) es la causa más común de la falla renal en el posoperatorio, resulta de manera primaria debido a la hipoxia de la médula renal (14). Los riñones reciben cerca de 20 a $25 \%$ del gasto cardiaco total en el adulto sano, cerca de $90 \%$ del flujo sanguíneo renal se dirige hacia la corteza, $10 \%$ restante hacia la médula donde se lleva a cabo aproxima- damente $90 \%$ de la extracción de oxígeno. La combinación de bajo flujo sanguíneo y alta demanda metabólica hace que la médula se vuelva muy susceptible a la hipoxia y a la insuficiencia renal durante y después de la cirugía (15).

El tono vascular intrarrenal depende del balance entre varios mediadores neurohumorales. Los mediadores vasodilatadores son el óxido nítrico, prostaglandinas, dopamina, adenosina, urodilatin, y los mediadores vasoconstrictores son la endotelina, angiotensina II y vasopresina. Durante la cirugía, la pérdida de fluidos conduce a isquemia renal con la consecuente disminución de la producción del óxido nítrico y la liberación de adenosina proveniente del consumo de trifosfatos de adenosina.

La adenosina causa vasoconstricción en la corteza renal y disminución de la FG con incremento en la reabsorción tubular renal. Este proceso activo causa aumento en los requerimientos de oxígeno medular, condicionando la aparición de IRA (16).

Los factores predisponentes para el desarrollo de IRA se pueden dividir en factores preoperatorios e intraoperatorios (17):

- Los factores preoperatorios incluyen la presencia de disfunción renal previa, diabetes mellitus (DM), disfunción cardiaca, edad > 65 años, sepsis, falla hepática, trauma y exposición a medicamentos o sustancias nefrotóxicas (18).

- Los factores intraoperatorios como hipovolemia, embolismo, isquemia renal e inflamación llevan a isquemia e incremento de la presión intraabdominal, y por último a IRA.

La anestesia, en especial los agentes volátiles, producen vasodilatación periférica y depresión miocárdica, favoreciendo la caída en la presión de perfusión y la posterior aparición de IRA. El uso de inhibidores de la enzima convertidora de angiotensina (IECA) puede ocasionar disminución significativa en la presión de perfusión renal, debido a la incapacidad en el mantenimiento de la vasoconstricción de la arteriola eferente (19). La presión intraabdominal (normal: de 0 a $17 \mathrm{mmHg}$ ), se puede incrementar por sangrado intraabdominal, distensión, peritonitis, íleo o ascitis; valores $>20$ $\mathrm{mmHg}$, ocasionan reducción de $75 \%$ de la FG y $>40 \mathrm{mmHg}$ son causa de anuria, siendo indispensable la descompresión urgente para mejorar las presiones de perfusión esplácnica y renal (20).

En los EE.UU. se realizan cerca de 600000 cirugías de revascularización miocárdica (RVM) cada año. La frecuencia de IRA en el posoperatorio es alrededor de 0.7 a $31 \%$ $(21,22)$.

Múltiples estudios han mostrado que los pacientes que van a CVC tienen flujo sanguíneo renal alterado, incremento en la resistencia vascular renal y FG disminuida $(23,24)$.

Mangano y colaboradores (25) realizaron un estudio prospectivo observacional con 2843 pacientes que fueron a RVM, se encontró que la incidencia de IRA en el posoperatorio fue de $7.7 \%$, de los cuales $1.4 \%$ requirieron diálisis. 
Estos resultados fueron similares a los publicados por Chertow y colaboradores, quienes evaluaron 43643 pacientes y la incidencia de IRA con requerimiento de diálisis fue de $1.1 \%$ y la mortalidad global fue $19 \%$, siendo de $69 \%$ en los pacientes que requirieron TRR (26).

Otros factores de riesgo para el desarrollo de IRA en el posoperatorio incluyen disfunción renal preexistente, género femenino, falla cardiaca, enfermedad cardiovascular preexistente, uso de balón intraaórtico, enfermedad pulmonar obstructiva crónica (EPOC), DM insulinodependiente, cirugía cardiaca previa, requerimiento de CEC y cirugía de la válvula mitral (27).

La aparición de IRA posterior a la CVC se asocia con incremento en los días de estancia hospitalaria, costos en salud y mortalidad (28).

La mortalidad de la IRA en CVC es de 9.5 a 100\% (29). La disfunción renal previa, el requerimiento de TRR en el posoperatorio, la disfunción multiorgánica, el tipo de cirugía y el compromiso aórtico (disección o ruptura aneurismática), se encuentran dentro de las causas más frecuentes de compromiso renal.

Zanardo y colaboradores (29), en un estudio prospectivo de 775 pacientes sometidos a CVC, observaron aumento de la mortalidad (17.1\% vs $3.4 \%$ ) y de la morbilidad en aquellos pacientes con creatinina preoperatoria $>1.5 \mathrm{mg}$ / $\mathrm{dL}$ con respecto a los pacientes que tenían un valor inferior.

La enfermedad renal crónica (ERC) previa (con o sin diálisis) incrementa tres a cuatro veces la mortalidad en el posoperatorio de CVC, aumentando el riesgo de mediastinitis, accidentes cerebrovasculares, riesgo de transfusión, tiempo de ventilación mecánica, prolongación de estancia hospitalaria; además, la ERC predispone a requerir soporte de diálisis de manera permanente y disminuye la supervivencia a largo plazo $(30,31)$.

El paciente con IRA en el posoperatorio de CVC puede cursar con disfunción multiorgánica, lo cual incrementa de forma dramática la mortalidad en $90 \%$ de los casos (39). Estos pacientes se encuentran con severa inestabilidad hemodinámica, falla respiratoria, inadecuado manejo de volumen intra y extravascular, anomalías hidroelectrolíticas y del estado ácido base y alteraciones de la coagulación (40).

Cada vez se hace más énfasis en identificar pacientes con factores de riesgo para IRA con el fin de implementar medidas terapéuticas adicionales para prevenir o disminuir la severidad de la disfunción renal.

En el desarrollo de modelos predictivos para la aparición de IRA, Chertow y colaboradores fueron los primeros en publicar una escala de riesgo para predecir aparición de IRA, usando 40000 pacientes a los que se les realizó RVM en 43 hospitales de los EE.UU. Los autores se basaron en los factores de riesgo reportados en la literatura para estratificar la IRA, encontrando deficiencias metodológicas inherentes de las cohortes como el número escaso de mujeres y pacientes afroamericanos (41). Sin embargo, esta escala fue la base para realización de otras que ofrecieron un mejor diseño y rendimiento diagnóstico.

Debido a las limitaciones de la escala previa, Thakar y colaboradores formularon y validaron otra escala (escala de Cleveland) para predicción de aparición de IRA con requerimiento de TRR. Utilizaron una cohorte de 33217 pacientes en la Clínica de Cleveland entre 1993 y 2002. Esta escala evaluó 13 factores de riesgo con significancia estadística en la aparición de IRA, los rangos oscilaban entre 0 y 13 puntos, los pacientes fueron clasificados en tres grupos de riesgo. El grupo de bajo riesgo ( 0 a 2 puntos) tuvo riesgo de requerir TRR de $0.4 \%$ y el grupo de alto riesgo ( 9 a 13 puntos) tiene riesgo de requerimiento de TRR de $21.5 \%$ (42).

Mehta y colaboradores, publicaron otra escala para evaluar el riesgo preoperatorio de requerir TRR después de CVC, evaluaron 449524 pacientes; la escala tuvo limitaciones debido al uso de 30 variables que ocasionaban dificultades para el adecuado uso clínico (43).

En la búsqueda de un modelo predictivo adecuado, Wijeysundera y colaboradores desarrollaron el Índice Renal Simplificado (IRS) para estimar el riesgo de requerir TRR después de CVC (escala de Canadá). Se evaluaron 20131 pacientes en Ottawa y Toronto, los cuales iban a CVC. Se establecieron ocho predictores para el desarrollo de la escala como FG entre 30 y $60 \mathrm{~mL} / \mathrm{min}$ y $<30 \mathrm{~mL} / \mathrm{min}$, DM, FEVI $<40 \%$, cirugía cardiaca previa, RVM acompañada de otro procedimiento, cirugía de urgencia y uso de balón de contrapulsación intraaórtico en el preoperatorio. La escala fue validada con dos cohortes, una de 2566 pacientes que fueron a RVM en el Hospital General de Toronto y otra cohorte de 6814 pacientes en el Instituto del Corazón de Ottawa entre 2004 y 2005. La escala tuvo limitaciones en el análisis debido a que no se pudieron establecer pacientes de riesgo moderado. Estratificaron los pacientes que obtuvieron más de cuatro puntos en pacientes de alto riesgo, lo cual comparado con modelos previos, se traduce en un riesgo de requerimiento de TRR de $10 \%$ (44).

Las ventajas de usar este modelo predictivo de riesgo para desarrollar IRA son la capacidad de discriminación por el número de variables, el uso práctico y la medición de la FG, comparado con modelos previos que sólo determinan la creatinina como método diagnóstico (45).

Estos modelos predictivos para determinar la aparición de IRA en el posoperatorio de CVC han facilitado la toma de decisiones, la evaluación y estratificación del riesgo, abriendo la posibilidad de realizar intervenciones más tempranas y efectivas en pro de la recuperación, disminución de complicaciones y descenso de la morbimortalidad en estos pacientes.

\section{Material y métodos}

La información fue recolectada por los investigadores a partir de la revisión de las historias clínicas, es decir, a partir de una fuente secundaria. Dicha recolección se realizó teniendo en cuenta un formulario diseñado para ese fin (Tabla 1), 
el cual incluye datos generales y datos de las variables de la escala de predicción:

- Se registraron los datos generales de cada uno de los pacientes, incluyendo antecedentes personales como historia de hipertensión arterial, DM y CVC.

- Se registraron los datos de las variables de la escala de predicción como el tipo de cirugía, intervención electiva o de urgencia, FEVI preoperatoria, FG preoperatoria calculada por la ecuación MDRD4, antecedentes como DM con requerimiento de medicación y la necesidad de balón de contrapulsación aórtica en el preoperatorio.

En pacientes que requirieron más de una intervención en la misma instancia, sólo se tomaron en cuenta los datos del primer episodio.

Durante la recolección de información se fue conformando la base de datos, la cual fue debidamente revisada y depurada, y posteriormente analizada utilizando el programa Epi Info 2000.

\section{Contexto y tipo de estudio}

Con el presente estudio se pretende describir el comportamiento de los pacientes en posoperatorio de CVC y el desenlace del requerimiento de TRR. Es un estudio observacional descriptivo, retrospectivo, tipo serie de casos.

- Identificar pacientes de alto riesgo de presentar injuria renal aguda en el posoperatorio de cirugía cardiovascular en la unidad de cuidados intensivos del Hospital Universitario San Ignacio.

- Establecer la frecuencia de presentación de los factores de riesgo descritos para el desarrollo de injuria renal aguda en el posoperatorio de cirugía cardiovascular.
- Identificar la incidencia de injuria renal aguda en pacientes de posoperatorio de cirugía cardiovascular en la unidad de cuidados intensivos del Hospital Universitario San Ignacio.

- Determinar la incidencia de requerimiento de terapia de reemplazo renal en pacientes en posoperatorio de cirugía cardiovascular.

- Comparar las incidencias de injuria renal aguda en el posoperatorio de cirugía cardiovascular en nuestro hospital respecto a lo escrito en la literatura médica.

\section{Criterios de ingreso y exclusión}

La población de referencia estuvo conformada por aquellos pacientes atendidos en el Hospital San Ignacio de la ciudad de Bogotá, institución de IV nivel de complejidad, con una población estudio conformada por aquellos pacientes que ingresaron a la unidad de cuidados intensivos en posoperatorio inmediato de cirugía cardiovascular. Debido a que se incluyeron todos los pacientes que cumplieron con los criterios de selección establecidos, no se calculó tamaño muestral.

\section{Criterios de inclusión}

- Pacientes adultos mayores de 18 años, de ambos sexos.

a Pacientes sometidos a revascularización miocárdica, reemplazo valvular, colocación de injerto en aorta con o sin bomba de circulación extracorpórea, trasplante cardiaco y biopsias pericárdicas.

\section{Criterios de exclusión}

- Pacientes con enfermedad renal crónica que están en hemodiálisis o diálisis peritoneal.

Tabla 1. Variables del estudio.

\begin{tabular}{|c|c|c|c|c|}
\hline Variable & Descripción & Tipo de variable & Escala de medición & Valor \\
\hline Fecha de procedimiento & Día de realización de la cirugía. & Continua & De razón & $\begin{array}{l}\text { Número expresado } \\
\text { en } \mathrm{dd} / \mathrm{mm} / \mathrm{aa}\end{array}$ \\
\hline Edad & Tiempo transcurrido desde el momento del nacimiento. & Continua & De razón & $\begin{array}{l}\text { Número expresado } \\
\text { en años }\end{array}$ \\
\hline Género & Lo que parece diferenciar la identidad femenina de la masculina. & Discreta & Nominal, dicotómica & $\begin{array}{l}1=\text { Masculino } \\
2=\text { Femenino }\end{array}$ \\
\hline Filtración glomerular & $\begin{array}{l}\text { Volumen de fluido filtrado por unidad de tiempo desde los capilares } \\
\text { glomerulares renales hacia el interior de la cápsula de Bowman. }\end{array}$ & Continua & Intervalo & $\begin{array}{l}\text { Número expresado } \\
\text { en } \mathrm{ml} / \mathrm{min}\end{array}$ \\
\hline Diabetes mellitus & Hace referencia a si el paciente tiene o no diagnóstico de diabetes mellitus. & Discreta & Nominal, dicotómica & $\begin{array}{l}1=\mathrm{Si} \\
2=\mathrm{No}\end{array}$ \\
\hline $\begin{array}{l}\text { Fracción de eyección } \\
\text { (VI) menor del } 40 \%\end{array}$ & $\begin{array}{l}\text { Hace referencia a si el paciente tiene o no una fracción de eyección del VI } \\
\text { menor al } 40 \% \text {. }\end{array}$ & Discreta & Nominal, dicotómica & $\begin{array}{l}1=\mathrm{Si} \\
2=\mathrm{No}\end{array}$ \\
\hline Cirugía cardiaca previa & $\begin{array}{l}\text { Hace referencia a si el paciente tiene antecedente de cirugía cardiaca } \\
\text { previa. }\end{array}$ & Discreta & Nominal, dicotómica & $\begin{array}{l}1=\mathrm{Si} \\
2=\mathrm{No}\end{array}$ \\
\hline Cirugía no electiva & Hace referencia a si la cirugía del paciente fue electiva. & Discreta & Nominal, dicotómica & $\begin{array}{l}1=\mathrm{Si} \\
2=\mathrm{No}\end{array}$ \\
\hline Procedimiento adicional & Hace referencia a si al paciente se le practicó algún procedimiento adicional. & Discreta & Nominal, dicotómica & $\begin{array}{l}1=\mathrm{Si} \\
2=\mathrm{No}\end{array}$ \\
\hline $\begin{array}{l}\text { Uso de balón de } \\
\text { contrapulsación intraaórtico }\end{array}$ & $\begin{array}{l}\text { Hace referencia a si previo a la cirugía del paciente se utilizó balón de } \\
\text { contrapulsación. }\end{array}$ & Discreta & Nominal, dicotómica & $\begin{array}{l}1=\mathrm{Si} \\
2=\mathrm{No}\end{array}$ \\
\hline
\end{tabular}


- Pacientes con historia de trasplante renal.

- Pacientes en posoperatorio de cirugía cardiovascular en otra institución.

- Pacientes sometidos a procedimientos menores como colocación de cardiodesfibriladores, marcapasos y ventanas pericárdicas.

\section{Análisis estadístico}

Se hizo el análisis estadístico descriptivo de la totalidad de las variables incluidas en la base de datos. Dichas variables fueron expresadas en frecuencias absolutas y porcentajes (variables discretas) o con promedios y desviaciones estándar (variables continuas).

Se calculó la incidencia de IRA identificando aquellos pacientes que requirieron TRR y posteriormente se efectuó un análisis bivariado, se compararon aquellos pacientes que fueron sometidos a TRR con los que no la recibieron. Para identificar diferencias significativas en el comportamiento de las variables se utilizó el test de student (variables continuas) y la prueba de chi cuadrado (variables discretas).

La información fue recolectada por los investigadores a partir de la revisión de las historias clínicas, es decir, a partir de una fuente secundaria. Dicha recolección se realizó teniendo en cuenta un formulario diseñado para ese fin, el cual incluye datos generales y datos de las variables de la escala de predicción:

Se registraron los datos generales de cada uno de los pacientes, incluyendo antecedentes personales como historia de hipertensión arterial, DM y CVC.

Se registraron los datos de las variables de la escala de predicción como el tipo de cirugía, intervención electiva o de urgencia, FEVI preoperatoria, FG preoperatoria calculada por la ecuación MDRD4, antecedentes como DM con requerimiento de medicación y la necesidad de balón de contrapulsación aórtica en el preoperatorio.

En pacientes que requirieron más de una intervención en la misma instancia, sólo se tomaron en cuenta los datos del primer episodio.

Durante la recolección de información se fue conformando la base de datos, la cual fue debidamente revisada y depurada, y posteriormente analizada utilizando el programa Epi Info 2000.

\section{Resultados}

Se analizaron 213 pacientes que ingresaron a la unidad de cuidado intensivo del Hospital Universitario San Ignacio en el posoperatorio inmediato de CVC, de los cuales $59.6 \%$ pertenecían al género masculino $(\mathrm{n}=127)$ y $40.4 \%$ al género femenino $(n=86)$. El promedio de edad observado fue 63.5 años con una desviación estándar de 12.3 años, encontrando pacientes entre 18 y 86 años. Entre las pacientes del género femenino la edad promedio fue $62.67 \pm 13.43$ años, siendo muy similar al encontrado entre aquellos del género masculino (62.64 \pm 11.4 años $)(\mathrm{p}=0.216)$.
Entre los pacientes analizados la incidencia de IRA fue $3.3 \%(\mathrm{n}=7)$ los cuales fueron sometidos a TRR. Estos pacientes tuvieron una creatinina posterior al procedimiento significativamente más alta $(\mathrm{p}=0.000)$ (Tabla 2$)$.

Entre los pacientes que requirieron TRR se encontró una proporción significativamente mayor de casos con una FEVI menor a $40 \%$ (71.4\% vs $14.1 \%$ ) (Figura 2) (Tabla 1).

Aunque no hubo diferencias significativas con relación al promedio de la FG entre los pacientes sometidos a TRR y aquellos que no la requirieron, sí se evidenció un porcentaje más elevado de pacientes con una FG mayor a 60 entre los pacientes que no requirieron TRR ( $77.2 \%$ vs $14.3 \%)$, y un porcentaje mayor de pacientes con FG entre 40 y 60 entre los pacientes que requirieron TRR $(57.1 \%$ vs $21.8 \%)$, diferencias que fueron significativas desde el punto de vista estadístico ( $\mathrm{p}=0.000)$ (Tabla 2) (Figura 3).

Otra diferencia significativa fue el porcentaje de pacientes en quienes se utilizó el balón intraaórtico, el cual fue mucho mayor entre los pacientes que recibieron TRR $(\mathrm{p}=0,000)$ (Tabla 2) (Figura 4).

En cuanto a la relación entre la IRA y los procedimientos realizados a los pacientes se evidenció una mayor proporción de casos manejados con CEC dentro del grupo de pacientes que requirieron TRR; así mismo, la proporción de pacientes a quienes se les colocó balón intraaórtico fue significativamente mayor $(\mathrm{p}=0.000)$.

El promedio de creatinina sérica fue significativamente más elevado en el grupo de pacientes que requirieron TRR en

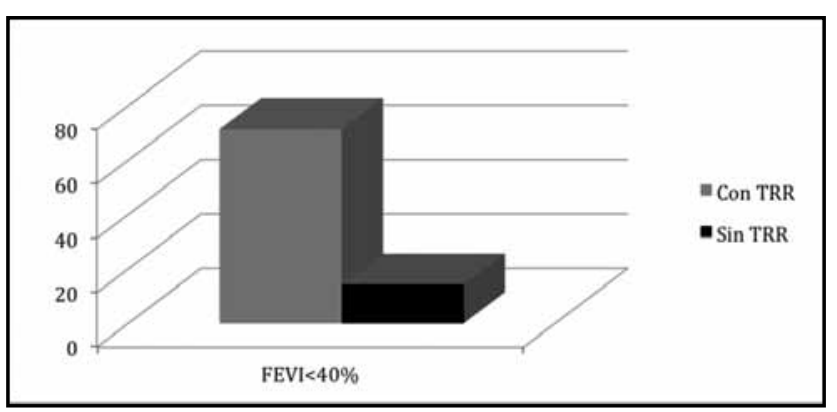

Figura 2. Porcentaje de pacientes con fracción de eyección del ventrículo izquierdo $<40 \%$ entre pacientes con y sin requerimiento de terapia de reemplazo renal.

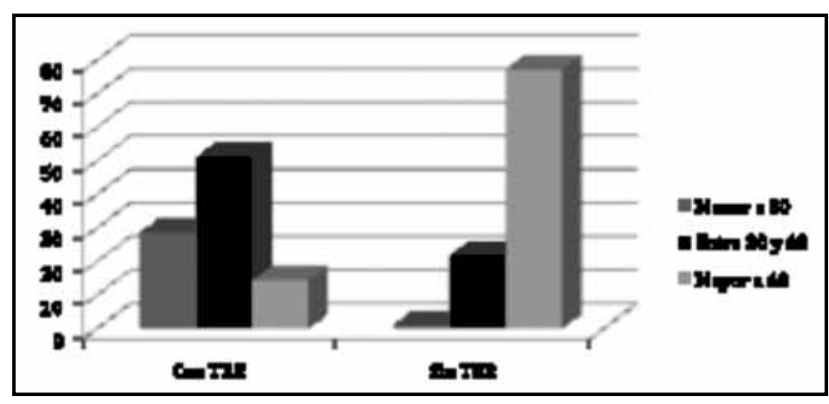

Figura 3. Distribución de los pacientes con y sin requerimiento de terapia de reemplazo renal (TRR) de acuerdo al comportamiento de la filtración glomerular. 
Tabla 2. Diferencias preoperatorias, perioperatorias y posoperatorias entre los pacientes que requirieron TRR y aquellos que no la requirieron.

\begin{tabular}{|c|c|c|c|c|}
\hline & $\begin{array}{c}\text { Total } \\
\text { de pacientes } \\
(n=213)\end{array}$ & $\begin{array}{c}\text { Pacientes } \\
\text { sin TRR* } \\
(\mathbf{n}=\mathbf{2 0 6})\end{array}$ & $\begin{array}{l}\text { Pacientes } \\
\text { con TRR* } \\
(n=7)\end{array}$ & $\begin{array}{l}\text { Valor } \\
\text { de p }\end{array}$ \\
\hline \multicolumn{5}{|l|}{ Género } \\
\hline Masculino & $127(59.6 \%)$ & $84(59.2 \%)$ & $2(28.6 \%)$ & 0.409 \\
\hline Femenino & $86(40.4 \%)$ & $122(40.8 \%)$ & $5(71.4 \%)$ & \\
\hline Edad (ds) años & $63.5(12.3)$ & $63.58(12.21)$ & $61.14(14.97)$ & 0.805 \\
\hline \multicolumn{5}{|l|}{ Antecedentes } \\
\hline Diabetes Mellitus & $32(15 \%)$ & $30(14.6 \%)$ & $2(28.6 \%)$ & 0.308 \\
\hline EPOC & $20(9.4 \%)$ & $19(9.2 \%)$ & $1(14.3 \%)$ & 0.652 \\
\hline Cirugías cardiacas previas & $8(3.8 \%)$ & $8(3.9 \%)$ & 0 & 0.595 \\
\hline \multicolumn{5}{|l|}{ FEVI menor $40 \% \dagger$} \\
\hline $\mathrm{Si}$ & $34(16 \%)$ & $29(14.1 \%)$ & $5(71.4 \%)$ & 0.000 \\
\hline No & & $177(85.3 \%)$ & $2(28.6 \%)$ & \\
\hline Filtración Glomerular & $78.8(28.1)$ & $79.4(25.5)$ & $62.11(72.6)$ & 0.109 \\
\hline \multicolumn{5}{|c|}{ Estratificación de la Filtración Glomerular } \\
\hline Menor a 30 & $4(1.9 \%)$ & $2(1 \%)$ & $2(28.6 \%)$ & 0.000 \\
\hline Entre 30 y 60 & $49(23 \%)$ & $45(21.8 \%)$ & $4(57.1 \%)$ & \\
\hline Mayor a 60 & $160(75.1 \%)$ & $159(77.2 \%)$ & $1(14.3 \%)$ & \\
\hline \multicolumn{5}{|l|}{ Procedimiento } \\
\hline Revascularización miocárdica & $98(46 \%)$ & $95(46.1 \%)$ & $3(42.9 \%)$ & 0.587 \\
\hline $\begin{array}{l}\text { Revascularización } \\
\text { miocárdica + válvula }\end{array}$ & $2(0.9 \%)$ & $2(1 \%)$ & 0 & 0.935 \\
\hline Circulación extracorpórea & $156(73.2 \%)$ & $150(72.8 \%)$ & $6(85.7 \%)$ & 0.448 \\
\hline Balón intraaórtico & $8(3.8 \%)$ & $6(2.9 \%)$ & $2(28.6 \%)$ & 0.000 \\
\hline Trasplante cardiaco & $9(4.2 \%)$ & $7(77.8 \%)$ & $2(28.6 \%)$ & 0.001 \\
\hline Otro procedimiento & $113(53.1 \%)$ & $102(96.5 \%)$ & $2(28.6 \%)$ & 0.825 \\
\hline \multicolumn{5}{|l|}{ Tipo de procedimiento } \\
\hline Urgencias & $30(14.1 \%)$ & $29(14.1 \%)$ & $1(14.3 \%)$ & 0.66 \\
\hline Electivo & $183(85.9 \%)$ & $177(85.9 \%)$ & $6(85.7 \%)$ & \\
\hline \multicolumn{5}{|l|}{ Tiempo de isquemia (ds) min } \\
\hline Total & $50.3(50.2)$ & $48.2(46.52)$ & $110.42(103.27)$ & 0.058 \\
\hline Trasplante cardiaco & $209.6(84.3)$ & $201.0(86.3)$ & 239.5 (99.7) & \\
\hline Otros procedimientos & $43.22(34.3)$ & $42.8(34.1)$ & $58.8(43.01)$ & \\
\hline Creatinina día 1 & $1.02(0.42)$ & $0.98(0.34)$ & $2.18(0.83)$ & 0.000 \\
\hline Creatinina día 3 & $1.01(0.66)$ & $0.92(0.02)$ & $3.54(0.48)$ & 0.000 \\
\hline \multicolumn{5}{|l|}{ Estado } \\
\hline Vivo & $196(91.6 \%)$ & $193(93.7 \%)$ & $2(28.6 \%)$ & 0.000 \\
\hline Muerto & $18(8.4 \%)$ & $13(6.3 \%)$ & $5(71.4 \%)$ & \\
\hline
\end{tabular}

el primer día posoperatorio, así como al tercer día $(\mathrm{p}=0.000)$. No se encontró ninguna diferencia en la creatinina del tercer día posoperatorio entre aquellos pacientes a quienes se les colocó bomba de CEC $(1.00 \pm 0.05)$ y aquellos que no la requirieron $(1.03 \pm 0.09)$.

$\mathrm{Al}$ aplicar la escala de riesgo en los pacientes analizados se evidenció que existían cuatro pacientes con alto riesgo de IRA cuyo puntaje fue mayor a cuatro. De estos pacientes solamente cuatro $(50 \%)$ terminaron en TRR.

\section{Discusión}

El manejo en cuidado intensivo del posoperatorio de la CVC, tiene como objetivo primario la recuperación de la homeostasis que se ve afectada por los cambios fisiopatológicos generados por el uso de la CEC. Los fenómenos de isquemia y reperfusión cardiaca y renal, la hipotermia, trastornos del sistema de coagulación, el sangrado y los efectos de las transfusiones, constituyen efectos deletéreos que tienen impacto negativo en el pronóstico y la evolución de la IRA. Se han analizado múltiples escalas de predicción de IRA, entre ellas la escala simplificada de riesgo (ISR) perioperatorio de requerimiento de TRR, siendo una herramienta sensible para predicción y detección de pacientes susceptibles de requerimiento de TRR en el posoperatorio de CVC.

La escala de Canadá en comparación con otros índices de predicción como la de Thakar et al y Mehta et al, muestra una mayor facilidad en su uso, debido al menor número de variables, la exactitud y precisión en su información. La escala usa ocho variables clínicas, lo cual favorece la estratificación de riesgo preoperatorio de requerimiento de TRR.

Los pacientes de alto riesgo ( $>$ de 4 puntos), se pueden beneficiar de medidas preventivas más enérgicas contra la aparición de IRA, haciendo estricto control del hematocrito, cirugía sin bomba de circulación extracorpórea (Off Pump) y seguimiento cuidadoso con marcadores de daño renal.

La aplicación de la escala a la población permitió mostrar que variables preoperatorias como la FG y la FEVI tuvieron una mayor significancia estadística respecto a otras variables como DM, cirugía cardiaca previa o procedimientos de

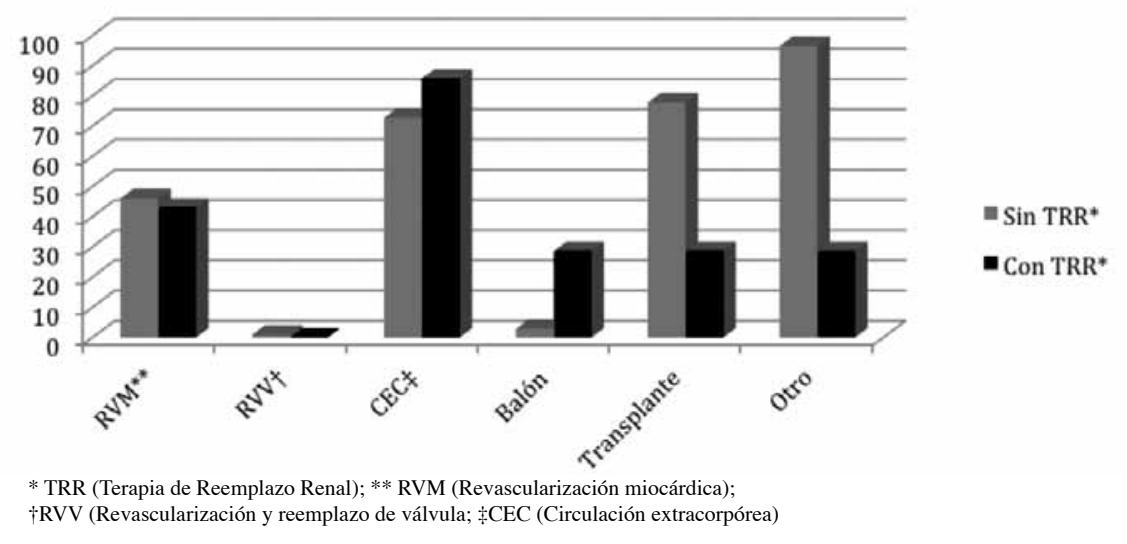

Figura 4. Procedimientos quirúrgicos realizados a los pacientes con o sin requerimiento de terapia de reemplazo renal. 
urgencia. En el intraoperatorio el uso de CEC y el tiempo de isquemia no tuvieron una significancia estadística en comparación con otras escalas como la escala de Cleveland (46). El uso de balón intraaórtico presentó significativa asociación con la aparición de IRA.

La incidencia de IRA en el posoperatorio de CVC en nuestro estudio fue de $3.3 \%$, similar a lo reportado en la literatura que es de 1 a 20\%. Escalas como la de Cleveland, encontraron que la realización de cirugías combinadas en especial en pacientes que iban a RVM y reparo valvular, presentaron un riesgo adicional para aparición de IRA, en nuestro estudio no encontramos una relación estadísticamente significativa $\mathrm{P}=0.9$.

La CVC sin el requerimiento de CEC ha mostrado ser una técnica quirúrgica segura y efectiva como lo demostró el metaanálisis de Wijeysundera y colaboradores (47), donde se vio una clara disminución en la mortalidad y aparición de complicaciones como la IRA en el grupo en que se realizó CVC sin CEC; en nuestro estudio no hubo diferencias significativas en la aparición de IRA, ni en el requerimiento de TRR con respecto al uso de CEC.

En escalas previas se había documentado la asociación estadísticamente significativa entre la DM y la IRA con requerimiento de TRR, en este estudio sólo se encontró en $28.6 \%$.

La tasa de mortalidad en los pacientes con IRA y requerimiento de TRR fue de $71.4 \%$, lo cual está en relación con lo reportado en la literatura.

En nuestro estudio tuvimos algunas limitaciones a considerar como que la institución no es un centro de referencia de CVC que nos permita obtener un mayor tamaño muestral. El número de pacientes manejados sin CEC fue bajo respecto al total de pacientes analizados en el estudio, lo cual dificulta la interpretación de los resultados y el impacto en la presencia de IRA en nuestra población.

\section{Referencias}

1. Chertow GM, Burdick E, Honour M, Bonventre JV, Bates DW. Acute kidney injury, mortality, length of stay, and costs in hospitalized patients. J Am Soc Nephrol 2005; 16: 3365-70.

2. Feest TG, Round A, Hamad S. Incidence of severe acute renal failure in adults: results of a community based study. BMJ 1993; 306: 481-33.

3. Thadhani R, Pascual M, Bonventre JV. Acute renal failure. N Engl J Med 1996; 334: $1448-60$.

4. Gailiunas P Jr, Chawla R, Lazarus JM, Cohn L, Sanders J, Merrill JP. Acute renal failure following cardiac operations. J Thorac Cardiovasc Surg 1980; 79: 241-3.

5. Conlon PJ, Stafford-Smith M, White WD, Newman MF, King S, Winn MP, Landolfo K. Acute renal failure following cardiac surgery. Nephrol Dial Transplant 1999; 14: 1158-62.

6. Mahon P, Shorten G. Perioperative acute renal failure. Curr Opin Anaesthesiol 2006; 19: 332-8.

7. Bellomo R, Ronco C, Kellum JA, Mehta RL, Palevsky P; Acute Dialysis Quality Initiative workgroup. Acute renal failure-definition, outcome measures, animal models, fluid therapy and information technology needs: the Second International Consensus Conference of the Acute Dialysis Quality Initiative (ADQI) Group. Crit Care 2004; 8: 204-12.

8. Kuitunen A, Vento A, Suojaranta-Ylinen R, Pettilä V. Acute renal failure after cardiac surgery: evaluation of the RIFLE classification. Ann Thorac Surg 2006; 81: 542-6.

9. Bove T, Calabrò MG, Landoni G, Aletti G, Marino G, Crescenzi G, et al. The incidence and risk of acute renal failure after cardiac surgery.$J$ Cardio thorac Vasc Anesth 2004; 18: 442-5.

10. Zanardo G, Michielon P, Paccagnella. A acute renal failure in the patient undergoing cardiac operation. Prevalence, mortality rate, and main risk factors. $J$ Thorac Cardiovasc Surg 1994; 107: 1489-95.

11. Svensson LG, Crawford ES, Hess KR, Coselli JS, Safi HJ. Variables predictive of outcome in 832 patients undergoing repairs of the descending thoracic aorta. Chest 1993; 104: 1248-53.

12. Svensson LG, Crawford ES, Hess KR, Coselli JS, Safi HJ. Experience with 1509 patients undergoing thoracoabdominal aortic operations. J Vasc Surg 1993; 17: $357-68$.

13. Weinstein GS, Rao PS, Vretakis G, Tyras DH. Serial changes in renal function in cardiac surgical patients. Ann Thorac Surg 1989; 48: 72-6.

14. Sheridan AM, Bonventre JV. Cell biology and molecular mechanisms of injury in ischemic acute renal failure. Curr Opin Nephrol Hypertens 2000: 9: 427-34 .

15. Brezis M, Rosen S. Hypoxia of the renal medulla Its implications for disease. $N$ Engl J Med 1995; 332: 647-55.

16. Conger JD. Vascular alterations in acute renal failure: Roles in initiation and maintenance.In : Acute Renal Failure - A Companion to Brenner and Rector's TheKidney, edited by Molitoris BA, Finn WF, Philadelphia, Saunders, 2001.p.13-29.

17. Jones DR, Lee HT. Perioperative renal protection. Best Pract Res Clin Anaes thesiol 2008; 22: 193-208.

18. Lok CE, Austin PC, Wang H, Tu JV. Impact of renal insufficiency on short and long term outcomes after cardiac surgery. Am Heart $J$ 2004; 148: 430-8.

19. Comfere T, Sprung J, Kumar MM, Draper M, Wilson DP, Williams BA, et al. Angiotensin system inhibitors in a general surgical population. Anesth Analg 2005; 100: 636-44.

20. Morken J, West MA. Abdominal compartment syndrome in the intensive care unit. Curr Opin Crit Care 2001; 7: 268-74.

21. Andersson LG, Ekroth R, Bratteby LE, Hallhagen S, Wesslen O. Acute renal failure after coronary surgery: A study of incidence and risk factors in 2009 consecutive patients. J Thorac Cardiovasc Surg 1993; 41: 237-41.

22. Bove T, Calabrò MG, Landoni G, Aletti G, Marino G, Crescenzi G, et al. The incidence and risk of acute renal failure after cardiac surgery. $J$ Cardio thorac Vasc Anesth 2004; 18: 442-5.

23. Rosner MH, Okusa MD. Acute kidney injury associated with cardiac surgery Clin J Am Soc Nephrol. 2006; 1: 19-32.

24. Carmichae IP, Carmichael AR. Acute renal failure in the surgical setting. ANZ J Surg 2003; 73: 144-53.

25. Mangano CM, Diamond stone LS, Ramsay JG, Aggarwal A, Herskowitz A, Mangano DT. Renal dysfunction after myocardial revascularization: Risk factors, adverse outcomes, and hospital resource utilization. Ann Intern Med 1998; 128: 194-203.

26. Chertow GM, Burdick E, Honour M, Bonventre JV, Bates DW. Acute kidney injury, mortality, length of stay, and costs in hospitalized patients. J Am Soc Nephrol 2005; 16: 3365-70.

27. Mangos GJ, Brown MA, Chan WY, Horton D, Trew P, Whitworth JA. Acute renal failure following cardiac surgery: Incidence, outcomes and risk factors. Aust NZJ Med 1995 25: 284-9.

28. McCullough PA, Wolyn R, Rocher LL, Levin RN, O’Neill WW. Acute renal failure after coronary intervention: incidence, risk factors, and relationship to mortality. Am J Med 1997; 103: 368-75.

29.Zanardo G, Michielon P, Paccagnella. A acute renal failure in the patient undergoing cardiac operation. Prevalence, mortality rate, and main risk factors. $J$ Thorac Cardiovasc Surg 1994; 107: 1489-95.

30. Liu JY, Birkmeyer NJ, Sanders JH, Morton JR, Henriques HF, Lahey SJ et al. Risk of morbidity and mortality in dialysis patients undergoing coronary artery bypass surgery. Northern New England Cardiovascular disease study group. Circulation 2000; 102: 2973-7.

31. Hirose H, Amano A, Takahashi A, Nagano N. Coronary artery bypass grafting for patients with non dialysis - dependent renal disfunction. Eur J cardiothorac Surg 2001; 20: 565-72.

32. Lameier NH. Contrast-induced nephropathy-prevention and risk reduction. Nephrol Dial Transplant 2006; 21: 11-23.

33. Meharwal ZS, Mishra YK, Kohli V, Bapna R, Singh S, Trehan N. Off pump multivessel coronary artery surgery in high risk patients. Ann Thorac Surg 2002; 74: 1353-7.

34. Jones DR, Lee HT. Perioperative renal protection. Best Pract Res Clin Anaesthesiol 2008; 22: 193-208.

35. Kashyap VS, Cambria RP, Davison JK, L'Italien GJ. Renal failure after thoraco abdominal aortic surgery. J Vasc Surg 1997; 26: 949-55. 
36. Mercatello A. Changes in renal function induced by anesthesia. Ann Fr Anesth Reanim 1990; 9: 507-24

37. Koning HM, Leusink JA, Nas AA, van Scheyen EJ, van Urk P, Haas FJ, et al. Renal function following open heart surgery: the influence of postoperative artificial ventilation. Thorac Cardiovasc Surg 1988; 36: 1-4.

38. Mangano DT. Aspirin and mortality from coronary bypass surgery. $N$ Engl J Med 2002; 347: 1309-17.

39. McCullough PA, Wolyn R, Rocher LL, Levin RN, O'Neill WW. Acute renal failure after coronary intervention: incidence, risk factors, and relationship to mortality. Am J Med 1997; 103: 368-75.

40. Faulı' A, Gomar C, Campistol JM, Alvarez L, Manig AM, Matute P. Pattern of renal dysfunction associated with myocardial revascularization surgery and cardiopulmonary bypass. Eur J Anaesthesiol 2005 20: 443-50.

41. Chertow GM, Lazarus JM, Christiansen CL, Cook EF, Hammermeister
KE, Grover F, et al. Preoperative renal risk stratification. Circulation 1997; 95: 878-84.

42. Thakar CV, Arrigain S, Worley S, Yared JP, Paganini EP. A clinical score to predict acute renal failure after cardiac surgery. J Am Soc Nephrol 2005; 16: 162-8.

43. Mehta RH, Grab JD, O'Brien SM, Bridges CR, Gammie JS, Haan CK, et al. Bedside tool for predicting the risk of postoperative dialysis in patients undergoing cardiac surgery. Circulation 2006; 114: 2208-16.

44. Wijeysundera DN, Karkouti KM, Dupuis JY, et al. Derivation and validation of a simplified predictive index for renal replacement therapy after cardiac surgery. JAMA 2007; 297: 1801-9.

45. Noyez L, Plesiewicz I, Verheugt FW. Estimated creatinine clearance instead of plasma creatinine level as prognostic test for postoperative renal function in patients undergoing coronary artery bypass surgery. Eur J Cardio thorac Surg 2006; 29: 461-5. 